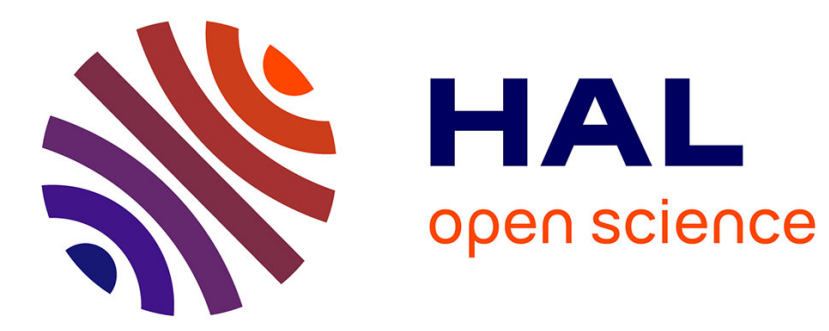

\title{
Sur la structure et les propriétés des rayons magnéto-cathodiques dans un champ uniforme \\ M. Gouy
}

\section{To cite this version:}

M. Gouy. Sur la structure et les propriétés des rayons magnéto-cathodiques dans un champ uniforme. Radium (Paris), 1911, 8 (4), pp.129-134. 10.1051/radium:0191100804012900 . jpa-00242461

\section{HAL Id: jpa-00242461 https://hal.science/jpa-00242461}

Submitted on 1 Jan 1911

HAL is a multi-disciplinary open access archive for the deposit and dissemination of scientific research documents, whether they are published or not. The documents may come from teaching and research institutions in France or abroad, or from public or private research centers.
L'archive ouverte pluridisciplinaire HAL, est destinée au dépôt et à la diffusion de documents scientifiques de niveau recherche, publiés ou non, émanant des établissements d'enseignement et de recherche français ou étrangers, des laboratoires publics ou privés. 


\section{MÉMOIRES ORIGINAUX}

\section{Sur la structure et les propriétés des rayons magnéto-cathodiques dans un champ uniforme ${ }^{1}$ \\ Par M. GOUY \\ [Faculté des Seiences de Lyon. - Laboratoire de Physique].}

\section{Analyse du faisceau magnéto-cathodique.}

En recevant sur une très petite ouverture un pareil faisccau, qui ne montre aucune structure délinie, on peut isoler des filets de rayons où l'on reconnaìt l'enroulement hélicoïdal que forment les trajecloircs des électrons daus un champ magnétique.

L'expérience réussit bien en prenant pour cathode une boule de $6 \mathrm{~mm}$ de diamètre. L'anode est un cylindre métallique de $8 \mathrm{~cm}$ de diamètre, dont l'axe est perpendiculaire au champ magnétique. Une feuille de métal le divise en deux compartiments, dont l'un contient la cathode, et l'autre un écran de willémite. Une ouverture de $0^{\mathrm{mm}}, 6$ de diamitre, percée dans cette cloison, laisse passer un filet magnéto-cathodique, dont la trace sur l'écran est observéc et photographiée à travers une glace parallèle. L'axe de la chambre noire est perpendiculaire au champ magnétique, en sorte que, si l'on déplace l'écran seul, les images produiles sur lui restent au point et peuvent être photographiées sur la mème plaque, marquant ainsi la trajectoire et les modifications du filet soumis à l'étude.

Les effets observés dépendent de la position de la cathode ${ }^{2}$, du voltage $\mathrm{V}$ et du champ $\mathrm{I}$; c'est ce dernier qu'on peut faire varier le plus commodément. On peut classer ces effets comme il suit :

a) Il $\mathrm{y}$ a une suite de points $\mathrm{F}_{1}, \mathrm{~F}_{2}, \mathrm{~F}_{3} \ldots$ où le filet produit sur l'écran une tache de la dimension de l'ouverture, et dont les distances à l'ouverture forment une progression arithmétique ${ }^{5}$. Entre ces foyers, le filet forme un fuseau creux, qui se manifeste sur

1. Le; conditions de ces expériences sont telles en général que les rayons magnéto-cathodiques soient normalement formés, c'est-a-dire que la pression est inférieure à $0.1 \mathrm{~mm}$ et le chrmp supéricur à 700 gauss. Le courant est continu, fourni par une machine électrostatique ou par une batterie d'accumulateurs.

2. La ligne de force magnétique passant par le centre de louverture doit rencontrer la cathode sous une incidence très oblique, pour que les elfets décrits plus loin se produisent.

5 J'ai । 4 de ees foyers, les dimensions de l'appareil ne permettant pas darantage. Ils diminuent un peu de netteté en s'éloignant de louverture.

T. 8. l'écran par une circonférence lumineuse, à milieu obscur, qui a sa dimension maximum au milicu de l'intervalle des foyers, et diminue quand on se rapproche de l'un d'eux. Il est rare que la circonférence soit entièrement fermée.

La figure 1 représente ce cas $(a)$; la photographic est à l'échelle de $2: 1$. La llèche indique le sens où marchent les rayons. On distingue le $1^{\text {er }}$ et le 2e foyer, et les figures intermédiaires, qui se montrent ici ovalisées par la perspective et l'obliquité de

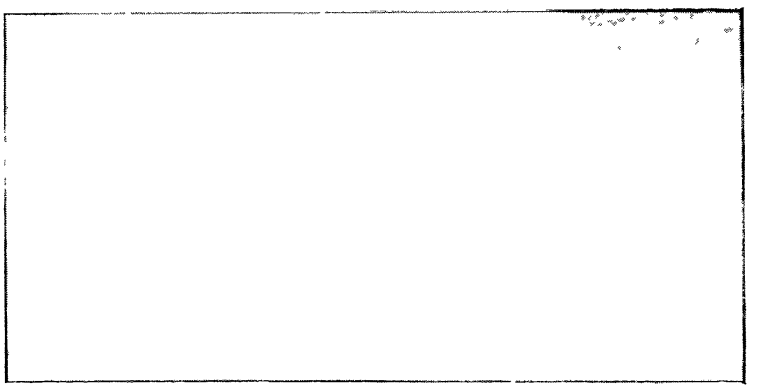

Fig. 1.

l'écran, mais sont circulaires sur l'écran quand il est normal aux rayons ${ }^{1}$.

b) Ces apparences se modifient en ce que la figure forme un croissant en se réduisant à un arc plus ou moins étendu, tel qu'une demi-circonférence par exemple, ce qui permet néanmoins de déterminer les foyers. Quelquefois aussi on a plusieurs taches lumineuses, qui se réduisent à une seule aux foyers.

c) Le filet est partout étroit, mais les taches successives sur l'écran dessinent une sinusoïde. Ce cas est le plus simple au point de vue de la discussion théorique, mais la sinusoïde étant très allongée, les expćriences sont moins démonstratives, et je m'en suis peu occupé.

1. Voici les donnèes de celte expérience : voltage 9500 : champ 1480. La distance de la cathorle à l'ouverture est d'environ $25 \mathrm{~mm}$, comme pour les autres expériences du tableau qui suit. 
Reprenons l'examen des cas $(a)$ et $(b)$. Soient $p$ l'intervalle des foyers consécutifs et $R$ le rayon de la circonférence dessinée sur l'écran au milieu de leur intervalle. Le tableau I donne les valeurs en millimètres.

Tableau I.

\begin{tabular}{|c|c|c|c|c|c|c|c|}
\hline r & II & l) & R & $1 \%$ & 11 & Callinde & Ca: ${ }^{1}$ \\
\hline $5200^{\prime}$ & $1470 \%$ & 9,5 & 》) & 10,3 & ) & loule $6^{\mathrm{mm}}$ & Air (courant). \\
\hline 6600 & 2180 & 7,1 & 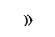 & 7,9 & ") & il. & id. \\
\hline 6800 & 1540 & 13,0 & $1, j$ & 12,7 & 12,0 & id. & II!̨drogine (eourant). \\
\hline 8500 & 1780 & 15,0 & 1,2 & 14,1 & 15,5 & id & id. \\
\hline $7: 00$ & 1520 & $10, \check{y}$ & 1,3 & 11,9 & $11, \overline{3}$ & ill. & id. \\
\hline 9050 & 2210 & 7,8 & 0,5 & 9,0 & 8,8 & id. & iil. \\
\hline 9500 & 1480 & 13,7 & 1,4 & $1 \overline{5}, 9$ & 13,2 & id. & id. \\
\hline 12800 & 1910 & $13, \tilde{3}$ & 1,5 & 12,5 & 11,6 & id. & Gaz de la calhode. \\
\hline 11200 & 1880 & 15,0 & 1,5 & 11,9 & 11.2 & id. & id. \\
\hline 11000 & $2 \vdots 90$ & 8,2 & 0,6 & 9,5 & 9.1 & id. & id. \\
\hline 9500 & 2500 & 8,5 & 0,6 & 9,0 & 8,7 & id. & id. \\
\hline 10100 & 1870 & 13,0 & 0,7 & 11,2 & 11,0 & id. & id. \\
\hline 5400 & 1070 & 14,0 & 1,3 & 14,5 & 13,9 & id. & Orygène. \\
\hline 4950 & 750 & 18,0 & 1,5 & 20,0 & 19,2 & id. & id. \\
\hline 5700 & 1050 & 16,5 & ) & 15,5 & ) & plaque $^{2}$ & IIydrogène (courant). \\
\hline 5760 & 1110 & $14, \check{5}$ & ) & 11,4 & ) & id. & id. \\
\hline
\end{tabular}

1. La pression était de $\varepsilon 0^{\mathfrak{\alpha}}$ à $40{ }^{i 2}$.

2. Plaque de $1 \mathrm{~cm}$ de còté, l'angle de la surface avec le champ élant $20^{\circ}$ à $50^{\circ}$. une rosace ayant son centre en 0 , et pour diamitre 2 ò. En général, quelques-unes seulement de ees trajectoires hílicoïdales rencontrent l'écran en 0 , mais en disposant de $\mathrm{V}$ et surtout de II, on arrive à les fairc passer toutes par l'ouverture, ce qui est facilité par ce fait que cette ouverture a des dimensions appréciables. Ilors, aux distances $\frac{p}{2}, \frac{5 p}{2}, \frac{5 p}{2}$, de l'ícran, ces trajectoires occuperont les points A situés sur la circonférence extérieure, et ces points $A$ en nombre infini dessincront une circonférence lumineuse de rayon o lou $\mathrm{R}$ du lableau $)^{1}$. Nous a vons donc au moins unc valeur moyenne de ó, si cette quantitć varic d'une hélice à l'autre d'une manière continue.

Les valeurs $p_{\mathbf{1}}$ du tableau ont été ainsi calculées; elles se rapprochent plus que $p_{0}$ des valeurs trouvées $p$, sauf

Examinons maintenant comment s'expliquent ces phénomènes dans la théorie électronique. La périodicité, qui en est le trait dominant, s'explique d'ellemême si les trajectoires d'électrons qui trarersent l'ouverture sont des hélices de même pas, car après un intervalle d'un pas ces trajectoires se trouveront dans les mèmes positions relatires. Calculons le pas $p$ de ces hélices, en admettant que les électrons partent sans vitesse de la surface de la cathode. Soient V la chute de potenticl du roint considéré jusqu’à la cathode, $v$ la vitesse de liélectron, $\theta$ la durée d'un tour autour de l'axe de l'hélice, $\delta$ le diamètre de celle-ci. Nous avons les équations.

$$
\frac{1}{2} m v^{2}=e V \quad, \quad 0=\frac{2 \pi}{\mathrm{H}} \cdot \frac{m}{e} \quad, \quad r^{2}=\left(\frac{p}{\theta}\right)^{2}+\left(\frac{\pi \grave{o}}{\theta}\right)^{2}
$$

d'où :

$$
p^{2}=\frac{8 \pi^{2} V}{H^{2}} \cdot \frac{m}{e}-\pi^{2} i^{2}
$$

Le tableau précédent donne les valeurs $p_{0}$ de $p$ calculées en supposant $\delta$ nul ' ; les valeurs de $p$ trouvées en diffèrent peu, ce qui est naturel, les hélices étant très allongées. Mais l'expérience elle-mème peut nous renseigner sur leur diamètre $\delta$.

Soit 0 l'ouverture (fig. 2), le plan de la figure étant normal au champ. Considérons toutes les hélices dont les projections passent au point 0 . Si elles ont toutes même diamètre $\delta$, ces projection s forment

$$
\text { 1. On prend } \frac{e}{m}=1,77 \cdot 10^{\circ} \text {. }
$$

ponr quelquis expériences laites arec $\mathrm{V}>10000$,

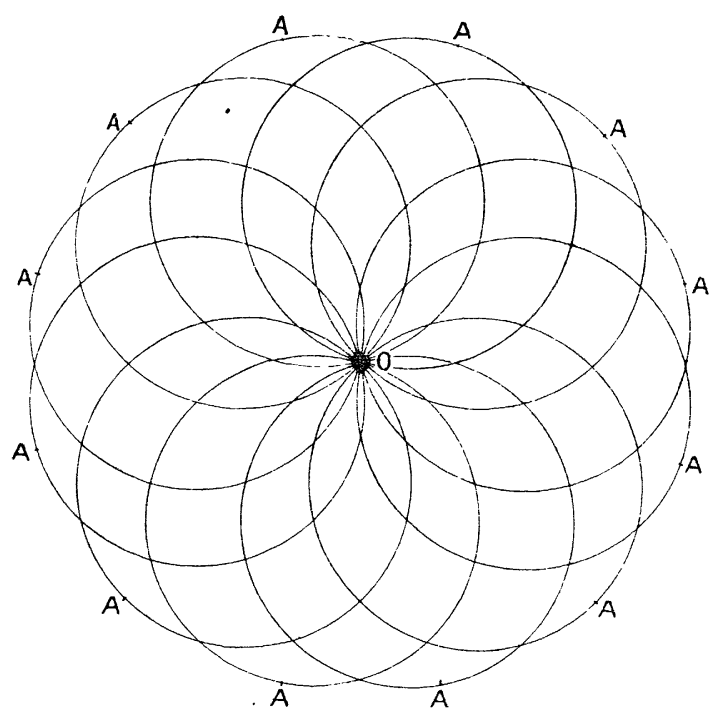

Fig. 2.

où les oscillatıons ont faussé quelque peu les rísultats ${ }^{2}$.

1. Ceci ne serait rigoureux que si le diamètre de l'ouverture était négligeable vis-à-vis de $\partial$. Mais l'erreur est corrigée en majeure partie, car $\mathrm{R}$ a été mesuré au milieu de l'épaisscur du trait brillant qui dessine la circonference, et eet aperçu sulfit au degré de précision des mesures.

2. On ne saurait trop insister sur la nécessité de se préoceuper, dans toutes les recherches de ce genre, des difficultés que créent les oscillations du courant. Quand les phénomènes dé- 
Il seraitintéressant de comparer $\delta$ avec des valeurs calculées à priori. Faute de micux, nous pourrons envisager deux cas extrèmes; $\alpha$ désignera l'angle que fait le champ magnétique avec la normale à la cathode.

$1^{0}$ Supposons que le champ électrique soit nul partout, sauf dans une couche infiniment mince à la surface de la cathode. On a :

$$
\delta=\frac{2 \sin \alpha}{I I} \sqrt{2 V \frac{m}{e}} .
$$

$2^{\circ}$ Supposons au contraire que le champ électrique soit partout uniforme. On a :

$$
\delta=2 \frac{m}{e} \frac{\operatorname{tg} \alpha}{H^{2}} \frac{V}{l}
$$

$l$ dísignant la distance du point considéré à la cathode, mesuréc sur la ligne de force magnétique.

Si l'on fait $V=10000, H=2000, x=45^{\circ}$ et $l=2 \mathrm{~cm}$, ces formules donnent $2^{\mathrm{mm}}, 4$ et $0^{\mathrm{mm}}, 14$. Les diamètres réels sont intermédiaires entre les valeurs calculées par les deux formules, et un peu plus voisins des premières.

\section{II. - Les caustiques des trajectoires des électrons.}

D'ordinaire, le sillon que trace dans le ga' le faisceau magnéto-cathodique ne montre pas de structure déterminíe, et ses bords sont un peu estompés, surtout dans des champs modérés et avec des voltages élerés. Cependant une structure très caractérisće cst visible dans des condilions favorables, it surtout au moyen d'un écran phosphorescent. : La tache lumineuse que produit le faiscéau sur l'écran, lorsque la cathode a une forme régulière, montre des bords très nets, et même dessinés par un trait plus brillant que le fond; d'autres lignes brillantes se montrent aussi dans l'intérieur de la tache, et varient avec la position de l'écran, le champ magnétique et le roltage.

Prenons, par exemple, pour cathode une boule de $8 \mathrm{~mm}$ de diamètre, placée à $7 \mathrm{~cm}$ environ de la paroi de verre, distance mesurée sur une ligne de force

pendent du potentiel, ce qui est ordinaire, elles produisent uu trouble, puisque l'on observe une superposition d'eflets correspondant à des potenticls différents, et il n'est pas nécessaire pour cela rutil y ait des oscillations, de simples variations suffisent. Mais. dans bien des cas, le régime oscillatoire, quand il s'útablit, correspond à un état de choses nouveau, qu'on ne peut rapprocher des phénomènes normaux. Cette difficulté empèche d'ordinaire d'utiliser les voltages supéricurs à 10000 . pour lesquels les oscillations sont difficiles à éviter; elle exige une surveillance attentive, soit au moyen du téléphone, soit en examinant l'aspect de la decharge. oì les nscillations se laissent toujours reconnaitre. magnéticue. Pour 150 gauss, on a sur le verre une tache de $20 \mathrm{~mm}$ à $50 \mathrm{~mm}$ de diamètre arec un point brillant en son centre. Le champ augmentant, ce point disprarait, et la ligne qui formait le contour extérieur se contracle et finit par former un point central pour 220 gauss. En mème temps, une autre circonférence brillante est apparuc, qui limite la tache, maintenant plus petite. Cette nouvelle ligne se contracte en son centre pour 280 gauss, ct ainsi de suite. Jusqu'à 1500 gauss, on voit ainsi les circonfërences apparaitre et se contracter, mais à la fin elles s'évanouissent arant d'avoir atteint le centre, et le milieu dela tache garde un éclat uniforme.

Lorsque le point central existe, il forme une sorte de foyer où concourt une assez notable partie du faisceau, car si en ce point on met un trìs petit écran, il en résulte une ombre qui couvre la majeure partie de la tache formée sur un écran plus éloigné.

En prenant pour cathode un fil cylindrique, on peut faire une étude plus détaillée du phénomène. sur un écran parallèle au fil, la tache forme une 
tres, de tolle sorte qu'elles présentent toutes leur pointe du côté où les rayons augmentent de longueur, leur bissectrices étant alignées suivant le milieu de la bande lumineuse (fig. 5) ${ }^{1}$.

Les linéaments qui les forment, étudiés à la loupe, n'ont souvent pas plus de $0^{\mathrm{mm}}, 1$ de largeur, et sont délicatement dessinés ${ }^{2}$. Les côtés convexes des V forment le contour extérieur de la bande lumineuse, et en dehors d'eux il n'y a aucune phosphorescence. La longueur du $\mathrm{V}$ et sa largeur diminuent quand le champ augmente, sans que la bande puisse jamais ètre moins large que la cathode. Si le diamètre de celte dcrnière est trop grand, les côtés du V ne se rejoignent plus, et par suite sa pointe n'est pas formée; la bande montre alors un éclat uniforme en son milicu, avec des côtés barbelés.

Si la cathode a un diamètre inférieur à $\mathbf{1}^{\mathrm{n} m}, \mathbf{3}$, les figures en $V$ commencent à montrer une distorsion qui s'accroît quand le diamètre diminue, et pour $0^{\mathrm{mm}}, 5$ elles ont une forme très compliquée. Il en est de mème quand on emploie, au lieu d'un fil cylindrique, une bande étroite, très oblique sur le champ.

Sans écran, cette structure périodique du faisccau magnéto-cathodique peut quelquefois être rue et

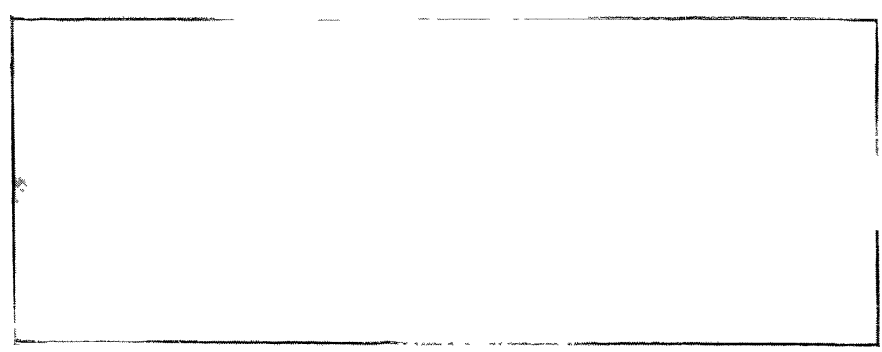

Fig 4 .

surtout pholographiée le long du sillon tracé dans le gaz, principalement dans l'espace obscur pré-cathodique. La figure 4 reproduit une photographie ${ }^{5}$ faite à l'échelle $2: 1$. On y distingue près de la cathode les figures en $\mathrm{V}$, analogues à celles qui se dessinent sur l'écran phosphorescent, et on constate que la cathode correspond à un maximum de largeur. Dans la partie brillante du faisceau, ces figures sont noyées dans une lumière d'une autre origine, dont nous reparlerons dans un moment.

1. Le cliché est à l'échelle de $5: 2$ environ. Le roltage est sensiblement $400^{\prime}$ ), le champ est de $1550 \mathrm{~g}$. et $2300 \mathrm{~g}$. La cathode, de $2 \mathrm{~mm}$ de diametre, est perpendiculaire au champ, et l'écran très incliné. Le's̀ nuages lumineux ne proviennent pas de l'écran, mais du gaz.

2. Une lame de verre (cristal) convient bien pour observer ces détails tris fins, et donne une lumière bleue propre à la photograpinie. Ces finesses se perdent en grande partie dans la reproduction.

3. Cathode de $1.5 \mathrm{~mm}$ de diamètre sur $6 \mathrm{~mm}$ de longueur, perpendıculaire au champ et parallele au rayon visuel. Voltage 5300. hamp $15 j 6$, courant 0.6 milliampère, pression $20 \%$. air.
L'explication de ces apparences doit évidemment être cherchée dans la manière dont les trajectoires des ćlectrons se concentrent en certaines portions de l'espace, en formant des caustiques tout à fait comparables aux caustiques des rayons lumineux, et qui sont, comme elles, les enveloppes des rayons individucls. La discussion ne peut en être faite, faute de connaitre exactement les trajectoires individuclles.

\section{III. - La lumière du faisceau magnéto-catho- dique est due surtout à un effet rétrograde.}

Reprenons l'expérience du chapitre I. Si l'obstacle (écran phosphorescent ou tout autre) arrête le filet magnéto-cathodique aux points où il forme les foyers $\mathbf{F}_{1}, \mathrm{~F}_{2}, \mathbf{F}_{5}$, le sillon lumineux tracé dans le gaz est une ligne fine, en réalité un petit cylindre qui a pour section la tache produite sur l'écran, tout le long de son parcours depuis l'ouverture, et même dans les régions où le filet forme un fuseau creux. Si au contraire le filet rencontre l'obstacle là où il forme fuseau, le sillon lumineux est large sur toute sa longueur, même là où le filet forme des foyers. Ce sillon n'est donc pas produit par l'illumination du gaz par les rayons incidents, mais consiste en un effet de retour, qui ne dépend que de l'état où se trouve le filet quand il rencontre l'obstacle où il se termine.

De même, quand on observe un faisceau magnéto-cathodique qui donne sur l'écran phosphorescent les figures en $\mathrm{V}$ (chap. II), on est surpris de ne pas voir celte structure si caractérisée se montrcr suivant la longueur du faisceau dans la partie brillante, en dehors de l'espace obscur pré-cathodique. A la vérité, la photographie en montre quelque chose dans des circonstances farorables, mais la presque totalité de la lumière dans cette région y est étrangère.

Voici du reste la preuve directe de cet effet rétrograde. Dans le cylindre-anode (fig. 5), plaçons une cathode formée d'un fil perpendiculaire au champ, qui est lui-même dans le plan de la figure. Le faisceau forme une nappe d'éclat sensiblement uniforme, depuis l'espace obscur jusqu'à l'anode. Si, dans cette nappe, on met un obstacle, comme un fil normal au plan de la figure et relié à l'anode, on roit ce fil former une ombre noire du côté opposé à la cathode, et une ombre grise du còté de celle-ci jusqu'ì l'espace obscur où elle disparait. Si, au lieu d'un fil, on prend une lame, on voit de même l'ombre noire $A$, et l'ombre grise B, mais celle-ci n'est obscure que si la lame est très inclinée sur les rayons; en deçà de $45^{\circ}$, la différence entre $B$ et le reste de la nappe est insensible, et on voit seulement deux lignes grises aux Jords de l'espace B. 
Il y a donc quelque chose qui revient de l'anode à la cathode en illuminant le gaz ${ }^{1}$; il est vraisemblable

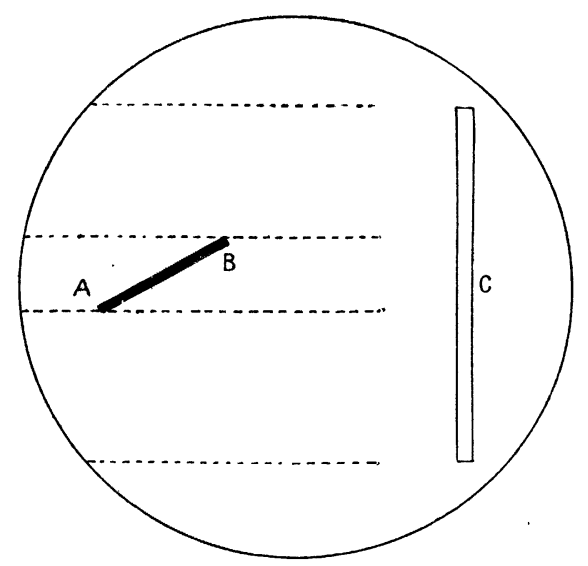

Fig. 5.

que ce sont les rayons cathodiques ajant subi la réflexion diffuse sur l'anode, qui reviennent en hélices serrées.

\section{IV. - Franges du faisceau magnéto-cathodique.}

L'effet rétrograde dont nous venons de parler est plus intense, et la lumière plus vive, quand l'obstacle où se termine le faisceau porte une charge négative, soit que celle-ci ait été apportée par le faisceau lui-même (cathode sccondaire), soit que l'ol;stacle soit une cathode auxiliaire. C'est un mode de décharge que j'ai nommé aclion inter-cathodique $^{2}$, et qui fera l'objet d'un autre travail. L'éclat lumineux de chaque filet du faisceau sur toute sa longueur, jusqu'à l'espace obscur, dépend alors, d'une manière souvent très marquée, de la phase où se trouve la structure périodique de ce filet au point où il rencontre l'obstacle. Sur la figure $\mathbf{3}$, on peut remarquer en effet que les petits nuages lumineux sont produits dans le gaz en des régions déterminées des figures en $\mathrm{V}$ tracées sur l'écran, qui paraissent correspondre à la plus grande concentration de la lumière phosphorescente.

De là résultent des franges longitudinales qui sillonnent le faisceau magnélo-cathodique, lorsque celui-ci forme une nappe un peu étendue. Ces franges sont parallèles au champ magnétique, et sont donc formées de rayons dont l'intensité lumineuse varie de l'un à l'autre suivant une loi périodique. On les produit aisément en faisant arriver le faisceau sur la paroi de

1. Il est clair que l'ombre grise B nous montre seulement la différence entre l'action de l'obstacle et celle de l'anode qu'il cache. J'ai pris pour obstacle des lames de platine, d'aluminium et de laiton sans constater une différence d'intensité bien sensible. L'ombre A est absolument noire, ne recevant ni rayons directs ni rayons rétrogrades.

2. Comptes rendus, 20 juin 1910. verre de l'ampoule (fig. 6) ${ }^{4}$; elles se produisent aussi très bien quand la cathode et l'obstacle sont deux fils situés dans un même plan, ce dermier élant à un potentiel inférieur de 100 volts environ à celui de l'anode. Enfin l'anode elle-même produit de pareilles franges, mais fort pâles, et qui ne seraient guìre visibles sans leurs mourements caractéristiques.

En effet, toutes ces franges, lorsqu'on fait varier l'intensité du champ magnétique, se déplacent régulièrement, et se succèdent les unes aux autres, en marchant avec des vitesses inégales, de telle sorte qu'elles se resserrent quand le champ augmente. Elles se comportent donc absolument comme font les franges d'interférences quand on passe du rouge au violet. Elles se déplacent aussi, à champ coustant, quand on fait varier la position de la cathode ou celle de l'obstacle.

Les mesures faites sur les clichís (particulièrement avec un fil de $1^{\mathrm{mm}}, 5$ ), montrent que les maxima correspondent à des rayons dont les longueurs sont en progression arithmétique, dont la raison $a$ parait proportionnelle à $\frac{\sqrt{V}}{H}$; pour 2000 volts et :000 gauss, on a sensiblement $7^{\mathrm{mm}}$. La longueur du rayon pour les franges brillantes a paru ètre un multiple entier de $a$, mais il n'en serait peut-être plus de mème pour d'autres cathodes. Quoi qu'il en soit, on en déduit 
probable que $a$ diminue un peu en se rapprochant de la cathode, et c'est ce que l'expérience semble confirmer.

Une variante de ces expériences consiste à réaliser une teinte plate en rendant les rayons d'égale longueur; elle réussit surtout avec la cathode auxiliaire mentionnée plus haut. Si l'on fait varier le champ magnétique, on voit l'éclat uniforme du faisccau varier périodiquement (saul dans l'espace obscur), el on peut ainsi compter une dizaine d'alternances entre 500 et 2000 gauss ${ }^{1}$.

\section{V. - Les rayons magnéto-cathodiques transportent des charges négatives.}

Lorsqu' un place dans le cylindre-anode de petites plaques reliées à l'anode, et telles que les rayons magnéto-cathodiques soient entièrement reçus par elles, on constate que ces plaques recueillent la presque totalité du courant; si les plaques sont isolées, elles s’élcctrisent négativement d'une manière tris apparente. Lorsqu'en tournant un peu l'ampoule, on fait tomber les rayons sur l'ano le, les plaques ne recucillent presque plus rien. Les rayons scrvent donc de véhicule au courant. Sur leur parcours, le gaz est assez fortement ionisé, comme on s'en assure au moyen de sondes ${ }^{2}$.

Toutefois, ce n'est pas par conduction, mais par convection que les rayons transportent le courant. M. Villard, M. Righi et M. Thirkill ont déjà étudić cette question, en recevant les rayons dans un cylindre de l'araday, sans obtenir d'indications nettes à l'électromètre, à causc, comme ils l'ont pensé avec raison, de la déperdition due à lionisation du gaz ambiant. Lne autre technique m'a donné, au contraire, une électrisation considérable. Pour diminuer la déperdition, le faisceau intense et de section très réduite est reçu sur une très petite lame de métal, émaillée au revers et sur ses còtés. Le cylindre-anode est divisé

données, on devrait avoir $9,4 \mathrm{~mm}$ pour une hélice de diamètre nul, $8,9 \mathrm{~mm}$ pour un diamètre de $1 \mathrm{~mm}$ el $7 \mathrm{~mm}$ pour un diamètre de $2 \mathrm{~mm}$. Mais icı le champ électrique n'est pas exactement counu, et la chute de potentiel est probablement plus petile que le voltage $\mathrm{V}$ de l'ampoule.

1. Voici un autre phénomène périodique, observé pendant ces expériences. Si l'on augmente le vide et le voltage jusyu à ce qu'on approche du point où il se produit spontanément un etat oscillatoire dans l'ampoule, on peut constater que cet élat s'élablit et cesse périodiquement une dizsine de fois. pendant que le champ varic de 500 à 2000 gauss. Pour un roltage trop faible, l'état oscillatoire ne s'établit pas; pour un voltage trop fort, il ne cesse pas. Ces oscillations sont rendues manifestes par le tiléphone at par le changement très visible qu'elles proiluisent dans la décharge.

2. Comples rendus, 15 mars 1909 . Hors du faisceau, le gaz s 1 moins contucleur el plus obseur ru'en l'absence du champ. en deux compartiments par une cloison de métal, percéc d'une fencitre garnic d'une toile très fine'. L'un de ces compartiments contient la cathode, et l'autre la lame. L'anode est au potentiel zéro.

Dans l'hydrogène, la lame, toujours négative, ne prend que quclques volts tant que la pression est sensiblement supérieure à 100 u. I.e vide augmentant, il se fait un changement assez brusque, et la lame prend plusieurs dizaines, puis plusicurs centaires de volts, jusqu’à 1000 v. ou 2000 r. dans certains cas, pour des champs de $2000 \mathrm{~g}$. el des vides de 20 y. Ce voltage varie dans le mème sens que le champ? ${ }^{2}$ Si l'on déplace qucligue peu la lame, de facon qu'elle ne reçoive plus le faisccau, son potentiel tombe à à quelques volts.

Dans l'air ou dans l'oxygìne, les effets sont du mème ordre, mais un peu moins considérables ${ }^{5}$.

Si l'on remplace la toile métallique par une leuille de métal percéc d'une ouverture de la dimension du faiscean, on a des effets un peu moins marqués, qui atteignent pourtant 400 volts avec l'hydrogìne '.

Les expériences de ce chapitre nous montrist que les rayons magnéto-callodiques transportent, par conrection, des charges négatives, ce qui est bien d'accord arec les autres phénomènes étudiés dans ce travail, qui ne peuvent s'expliquer qu'en admettant que ces rajons sont formés de trajectoires hélicoïdal $\mathrm{t}$ d'électrons. A ce point de rue, les mesures de la période sont tout à fait démonstratives. Les rayons magnélo-cathodiques sont donc formés de rayons cathodiques, mais on peut se demander si le champ magnétique ne donne pas aux électrons des propriétés nouvelles; l'expérience seule peut répondre. L'existence des franges, étudiées au chapitre IV, pourrait faire penser à une périodicité rappelant celle des accès de Yewton, mais peut-être sera-t-il possible d'en rendre comple plus sinıplement.

[Manuscrit reçu le 10 mars 1911].

1. La calhode est un fil de $1, \grave{3} \mathrm{~mm}$ de diamétre et $6 \mathrm{~mm}$ de longueur; la lame a $1 \mathrm{~mm}$ sur $4 \mathrm{~mm}$. La toile a 63 fils au centimètre; le courant est de 0,6 milliampère, et le champ de 500 à 2500 gauss La distance de la calhode à la toile est de $4 \mathrm{~cm}$ environ.

2. Si la lame est reliće a l'anode, elle recueille 10 a 15 pour cent du courant total de l'ampoule.

5. II. Thirkill mentionne que dans ses expériences, la pression a varié de $0.6 \mathrm{~mm}$ à $0,1 \mathrm{~mm}$ (Proc. Roy. Soc., mars 1910). ('est done l'insuffisanee du vide, jointe aux grandes dimensions du réecpteur cylindre de Faraday), qui a empèché l’électromitre de domer des indications sensibles.

4. I)ans ce cas, il se produit entre la lame el la calhode laclion inter-eathodique, qui fait baisser le voltage. La toile métallique diminue beaucoup cutte action, comme d'autres expériences l avaient montré, ce qui rend compte du voltage plus fort qu'elle permet d'ohtenir. 